\title{
Effectiveness of psychopharmacology in Anorexia Nervosa treatment
}

\author{
LUKASZ ZADKA
}

II Department of Psychiatry and Psychiatric Rehabilitation, Medical University of Lublin, 20-439 Lublin, Głuska 2, Poland

\section{ARTICLE INFO \\ Received 03 March 2015 \\ Accepted 07 April 2015}

\section{Keywords:}

anorexia nervosa,

treatment,

psychopharmacology.

\begin{abstract}
The eating disorder that generates the highest death rate is that of anorexia nervosa, and current treatment is a combination of equalization of somatic state and patient education. Moreover, psychical symptoms occurring in the course of anorexia nervosa are thought to have a crucial influence on the course of the disease. Hence, in medical literature, the effectiveness of psychotherapeutic interventions is also widely described. Still, the implementation of appropriate psychopharmacology is now considered an additional method of treatment, rather than a therapy of choice. Yet, in spite of many years of research, there are no absolute recommendations given, nor are instructions within the scope of psychopharmacological treatment proffered, although the selection of psychopharmacological items must respect both the patient's psychic and somatic states. In recent years, the popularity of psychopharmacological treatment has increased; therefore, we feel that it is justified to present the latest scientific information in this respect.
\end{abstract}

\section{INTRODUCTION}

Anorexia Nervosa (AN) is a disorder, in the course of which, there is usually a false body self-image generated, and there are several theories explaining the pathological grounds of anorexia nervosa. Some researchers consider AN as a disease entity, while others treated the disease as a pluricausal symptom complex. In the ICD-10 (International Classification of Diseases) classification, AN has been placed in the category of behavioral complexes related to specific physiological disorders. In current literature, two types of AN are defined: restrictive - when there is a self-established limitation of the eating of food; bulimic - when during any disease episode, there is alternating periods of binge eating followed by self-induced vomiting. Patients with the bulimic type of AN might also overuse purgatives and diuretics in order to bring about complete intestinal purge.

Currently, the dominating opinion is that AN comes about through a multi-factorial conditioning. Genetic, social-cultural, individual and family factors seem to be crucial in the disease pathogenesis, while life events and psychic trauma are also potential triggers for anorexia nervosa symptoms occurring.

\footnotetext{
* Corresponding author

e-mail: etamercury@gmail.com
}

The influence of emotional factors on appetite is fundamental to AN development, and the psychological features of AN play an important role in this pathogenesis. Many a time, AN patients reveal neurotic tendencies, lowered self-esteem and specific personality traits. Yet, it is hard to specify unambiguously whether the psychic symptoms occurring in the course of anorexia nervosa are of a primary or secondary character.

Kucharska-Pietura and colleagues emphasize the significant meaning of emotional processes in the pathogenesis of eating disorders. Moreover, Kouidrat and colleagues note that among the population of men diagnosed with anorexia nervosa, the co-diagnosis of schizophrenia occurs over three times more often than in women with an identical diagnosis. Furthermore, some of the false conviction characteristics for AN patients, including a deformed image of one's own body and fear of "being fat" - also are seen in people suffering from schizophrenia [11,15].

The course of AN is often adverse and might end with death, while the extreme eating patterns and eating addictions of patients with AN who display significant malnutrition, are the source for the several serious somatic symptoms that are manifested in various bodily systems and organs. Hospitalization is considered necessary for patients demonstrating extreme malnutrition. The bench-mark for such 
intervention is that values of Body Mass Index (BMI) are less than $15 \mathrm{~kg} / \mathrm{m}^{2}$, or the decrease of body mass is greater than $25 \%$ of the mass the individual should have. Moreover, significant life-threatening somatic disorders are evident, and suicide has been contemplated or attempted. Furthermore, there is a sequence of other, individual factors which should be considered by any specialist in evaluating the psycho-somatic condition of AN patients, when justifying hospitalization.

$\mathrm{AN}$ is notably seen among young women aged between 15 to 19 . It is also evident among males and among children of both sexes. Currently, the spread of this disease in the national population is not high, but it is growing. In Dutch and British research, the frequency of this disease in men amounts to $1 / 100000$ [12], while epidemiological data of this disease among paediatric patients, estimates its frequency to be within the scope of 1.4-2.6 of disease occurrence among 100000 people annually $[6,2]$.

The seriousness of the course of AN progression, and its high death rate have forced researchers to improve upon current therapeutic standards, and develop new therapies. At the moment, psychopharmacological treatment in cases of anorexia nervosa are seen as being only justified in specific situations, and to be implemented solely when the therapy applied so far has not brought about positive developments. Moreover, there must be no contraindications from the patient.

In AN psychopharmacotherapy, current knowledge is insufficient, and in spite of scientific progress, there are still no detailed, randomized researches conducted with the use of double-blind trials. Thus, an astute estimation of the latest medical research results concerning the psychopharmacological treatment of hospitalized AN patients is felt necessary.

\section{DESCRIPTIVE CHARACTERISTIC OF SELECTED DRUGS}

\section{Antidepressants}

Among An patients, significantly higher comorbidity with depressive disorders has been observed. Their depressed mood is often connected with a low BMI, and body mass compensation most frequently engenders a restored euthymia. The exhibited continuous mood disorders might be a recommendation to prescribe antidepressant drugs.

Current literature suggests that the application of selective serotonin reuptake inhibitors (SSRIs) might bring therapeutic advantages. Kaye and colleagues, by way of randomized researches and double-blind trials with the control application of placebos, have noted the effectiveness of fluoxetine administration. In this work, in the restrictive form of anorexia nervosa, an annual administration of fluoxetine has been seen to reduce the intensity of the disease symptoms, and has lead to an increase in body mass, thus, preventing a recurrence of AN [13].

Yet the results of such research has proved ambiguous. In the randomized research undertaken by Walsh and colleagues, the superiority of fluoxetine over a placebo was not confirmed [1]. It was however worth emphasizing that in the conducted examination, all patients/participants were also subjected to individual behavioral-cognitive therapy
(CBT), as, in case of adults, this is, currently, the most effective method of anorexia nervosa therapy. The application of CBT might explain the lack of effectiveness of fluoxetine over a placebo.

In the literature, it is known that some tricyclic antidepressants (TLPD) might increase appetite and body mass by potentiating the feeling of hunger. This kind of dependence was noticed in the case of clomipramine [8]. However, it should be remembered that the application of TLPD might be the reason for adverse reactions within the circulatory system. This effect, in AN patients, might have bad consequences. Therefore, the utility of this group of drugs had a limited application.

Strobel and colleagues have compared the effectiveness of clomipramine, with paroxetine, in treating young women co-diagnosed with anorexia nervosa and recurrent depression. Their work indicates the therapeutic effectiveness of both drugs. Of note: paroxetine therapy increased the values of BMI similarly to clomipramine administration, but the profile of demonstrated adverse reactions was more advantageous [5].

Tandospirone is an antidepressant of a strong anxiety reducing effect. This drug is a selective 5-HT1A receptor partial agonist, and literature suggests that it is effective in AN therapy. Long-term drug administration of tandospirone in persons with a restrictive and bulimic $\mathrm{AN}$, brought about an increase in their BMI [10]. More research, however, is necessary.

\section{Second-generation antipsychotics}

Olanzapine and risperidone are typical neuroleptics of a therapeutic effect in patients with AN that has been broached in scientific literature several times. One such study was conducted by Bissada and colleagues, who, in a randomized research study utilizing a controlled placebo, have demonstrated the effectiveness of olanzapine in bringing about a faster body mass increase, faster gain of expected BMI values, and in engendering a soothing of AN-related symptoms [4].

Regarding, risperidone, another study has shown that, following a 7 week course of administration of risperidone of a medium dose of $2.5 \mathrm{mg} / 24 \mathrm{~h}$, in adolescent AN patients, their placement results have been significantly lowered on the sub-scale Eating Disorder Inventory 2 Drive for Thinness. The effectiveness of the drug versus body mass increase, as well as the improvement of own-body perception was not noticed [16]. However, in a single case of a long-term administration of risperidone involving a 17 year old woman, a beneficial increase of body mass, and a decrease of obsessive thoughts intensity were noticed [3]. Furthermore, the effectiveness of risperidone administration in countering the effects of a restrictive form of AN was also documented in a 10 year old boy who had been prescribed olanzapine previously. The first improvement was noticed after a month of the drug administration [9].

Quetiapine is another neuroleptic which might positively influence AN therapeutic effects. In a randomized controlled trial, conducted by Court and colleagues, low doses of quetiapine were administered to young patients for a period of 6-12 months. The result of this work was that they put 
forward that the drug positively influenced the physical/ physiological functioning of the patients under treatment [14]. However, the work of Powers and colleagues, by way of a randomized study, were not able to confirm the superiority of quetiapine over a placebo [7].

\section{CONCLUSIONS}

Anorexia nervosa therapy should first consider the somatic state of the AN patient. Equalization of organism abnormalities and gained control of basic life parameters are a necessity among hospitalized patients. What is more, implementing early psychological interventions, as well as patient education within the scope of symptomatology, treatment and disease complications are equally important.

As of today, there are no specified standards for AN psychopharmacotherapy. Hence, due to the ambiguous results of conducted studies and the insufficient amount of data, it is crucial to undertake detailed, controlled randomized researches for this, involving placeboes, and to seek out new therapeutic possibilities which would increase the effectiveness of the treatment methods applied so far.

\section{REFERENCES}

1. Bissada H. et al.: Olanzapine in the treatment of low body weight and obsessive thinking in women with anorexia nervosa: a randomized, double-blind, placebo-controlled trial. Am J Psychiatry, 165, 10,2008.

2. Court A. et al.: Investigating the effectiveness, safety and tolerability of quetiapine in the treatment of anorexia nervosa in young people: a pilot study. J Psychiatr Res., 44, 15, 2010.

3. Hagman J. et al.: A double-blind, placebo-controlled study of risperidone for the treatment of adolescents and young adults with anorexia nervosa: a pilot study. J Am Acad Child Adolesc Psychiatry, $50,9,2011$.
4. Kaye W.H. et al.: Double-blind placebo-controlled administration of fluoxetine in restricting- and restricting-purging-type anorexia nervosa. Biol Psychiatry, 2001 49, 7, 2001.

5. Kouidrat Y. et al.: Eating disorders in schizophrenia: implications for research and management. Schizophr Res Treatment, 2014.

6. Kracke E.J., Tosh A.K.: Treatment of anorexia nervosa with longterm risperidone in an outpatient setting: case study. Springerplus, 2, 3, 2014.

7. Kucharska-Pietura K. et al.: The recognition of emotion in the faces and voice of anorexia nervosa. Int J Eat Disord., 35, 1, 2004

8. Lacey J.H., Crisp A.H.: Hunger, food intake and weight: the impact of clomipramine on a refeeding anorexia nervosa population. Postgrad Med J., 56, 1, 1980.

9. Madden S. et al.: Burden of eating disorders in 5-13-year-old children in Australia. Med J Aust., 20, 8, 2009.

10. Okita K. et al.: Tandospirone, a 5-HT1A partial agonist is effective in treating anorexia nervosa: a case series. Annals of General Psychiatry, 12, 7, 2013.

11. Pinhas L. et al.: Incidence and age-specific presentation of restrictive eating disorders in children: a Canadian Paediatric Surveillance Program study. Arch Pediatr Adolesc Med., 165, 10, 2011.

12. Powers P.S., Klabunde M., Kaye W.: Double-blind placebo-controlled trial of quetiapine in anorexia nervosa. Eur Eat Disord Rev., 20, 4, 2012.

13. Smink F.R., van Hoeken D., Hoek H.W.: Epidemiology of eating disorders: incidence, prevalence and mortality rates. Curr Psychiatry Rep., 14, 4, 2012.

14. Strobel M. et al.: Paroxetine versus clomipramine in female adolescents suffering from anorexia nervosa and depressive episode - a retrospective study on tolerability, reasons for discontinuing the antidepressive treatment and different outcome measurements. Z Kinder Jugendpsychiatr Psychother., 32, 4, 2004.

15. Umehara H., Iga J., Ohmori T.: Successful Treatment of Anorexia Nervosa in a 10-year-old Boy with Risperidone Long-acting Injection. Clin Psychopharmacol Neurosci., 12, 1, 2014.

16. Walsh B.T. et al.: Fluoxetine after weight restoration in anorexia nervosa: a randomized controlled trial. JAMA, 14, 22, 2006. 